Gut, 1973, 14, 773-777

\title{
The permeability of the small intestinal mucosa: A study using iron
}

\author{
C. A. LOEHRY, D. PARISH, AND JUNE BAKER
}

From the Royal Victoria Hospital, Bournemouth

SUMMARY Various substances containing iron were injected into the lumen of the small intestine in rabbits and into the mesenteric arterial supply. Sections of the mucosa were stained for ferric ions by immersing for 30 minutes into equal parts of $2 \% \mathrm{HCl}$ and $2 \%$ potassium ferrocyanide (Prussian Blue reaction). The ferric ion is bound to various cell structures and its passage through the mucosa could therefore be demonstrated. Iron appeared to pass from the blood into epithelial cells, especially near the tips of the villi, and also between cells as far as the junctional membrane. Studies using ${ }^{59} \mathrm{Fe}$ demonstrated the rapidity of transfer of iron from the blood into the intestinal lumen. After a period of ischaemia epithelial cells in other situations also appeared permeable to iron. The implications of the loss of substances from the blood into the lumen by these routes is discussed.

There is a continuous passage of substances from the blood out into the intestinal lumen. It has been demonstrated that mucosal permeation rate both in animals and man depends directly upon the plasma concentration of any substance and inversely upon its molecular size (Loehry, Axon, Hilton, Hider, and Creamer, 1970; Loehry, 1973). How these macromolecular substances actually permeate the intestinal mucosa is not fully understood; theoretically it is possible that they pass through epithelial cells, between them, or through gaps left by extruded cells at the tips of the villi. In order to study this further we injected substances into the arterial supply to the small intestine that could be directly visualized in the mucosa. In the present experiments we have used solutions containing iron as the ferric molecule becomes rapidly fixed to various mucosal and connective tissue constituents and is therefore not removed during fixing and staining procedures.

\section{Methods}

The experiments were performed on albino rabbits weighing between 2.5 and $3.5 \mathrm{~kg}$ under nembutal anaesthesia. The abdomen was opened by a midline incision and loops of small intestine were gently drawn out. Two-inch segments of intestine were clamped off and $0.25 \mathrm{ml}$ of various iron preparations

Received for publication 12 June 1973. injected directly into the mesenteric arterial supply to the segment. A biopsy was taken from the segment at a predetermined time interval and the tissue fixed in $10 \%$ formalin. No more than four segments were used in each animal and a control was always taken at the end of the experiments from a portion of bowel not used to ensure that there had not been any significant staining from iron that had leaked into the general circulation. In the experiments where the experimental segment was left for more than one minute after injection, the bulldog clamps isolating the segment were removed to prevent ischaemia. The mucosal biopsies were stained with $2 \% \mathrm{HCl}$ and $2 \%$ potassium ferrocyanide for 30 minutes (Perls' Prussian blue reaction) and equal parts of $20 \%$ potassium ferricyanide and $1 \%$ hydrochloric acid for 15 minutes (Turnbull's stain). Experiments were performed with solutions of ferrous and ferric sulphate. (4 $\mathrm{g}$ in $15 \mathrm{ml}$ saline), iron sorbitol/ascorbic acid (Jectofer), and irondextran (Imferon).

In another series of experiments the iron was injected into the loop intraluminally and biopsies were taken in a similar fashion.

In further experiments the loop was made ischaemic by clamping off the mesenteric arterial supply for periods up to 20 minutes before the intraarterial injection of ferrous sulphate.

In order to ascertain the distribution in the mucosa of iron staining substances $10 \mu$-thick sections were 
cut in a cryostat from fresh frozen mucosal biopsies and dipped into the same solutions of iron that had been injected. They were then washed in distilled water for 20 minutes before being fixed and stained as described.

To study the permeability of the intestinal mucosa to the iron molecule, $50 \mu \mathrm{c}$ of ${ }^{59} \mathrm{Fe}$ was injected directly into the mesenteric arterial supply to a 2-in segment of jejunum which was continuously perfused with normal saline at $40^{\circ} \mathrm{C}$ at a speed of $600 \mathrm{ml}$ per hour. Specimens were taken at 10 -second intervals up to 10.5 minutes and the activity was counted in a well type scintillation counter.

\section{Results}

INTRAARTERIAL IRON

Blue staining was obtained in a similar distribution with ferrous and ferric sulphate with both Perls' and Turnbull's stain. The former stain, however, gave the clearest results, presumably as it stains

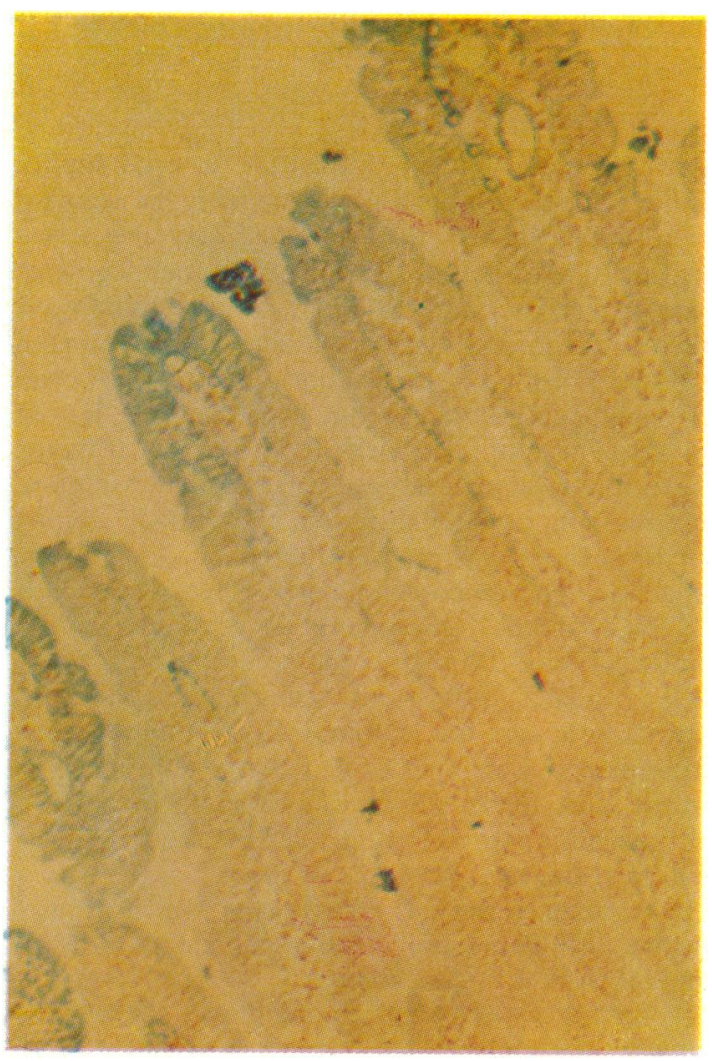

Fig 1 Perls' stain of jejunal mucosa one minute after the injection into the mesenteric artery of ferrous sulphate. $\times 200$. ferric ions and injected ferrous ions are rapidly converted by the mucosal cells to the ferric form. Within 10 seconds of the injection of ferrous and ferric sulphate, staining was observed in the epithelial cells at the very tips of the villi and in the basement membrane of the capillary network. Ferric ions also were shown at the junction of epithelial cells as far up as the tight junction and especially near the villous tips. At 30 seconds both substances showed marked staining in the cells at the tips of the villi, at the junction between cells, and in the capillary membrane. By one minute most of the villous cells showed some stain though it remained most marked in the areas mentioned. Only the occasional crypt cell showed staining. Figure 1 is a low-power view of the mucosa one minute after the injection of ferrous sulphate and demonstrates the preferential staining at the tips. Figure 2 is a higher magnification and shows iron in the capillary basement membrane, in the epithelial cells, especially near the tips and at the junctional layer. This

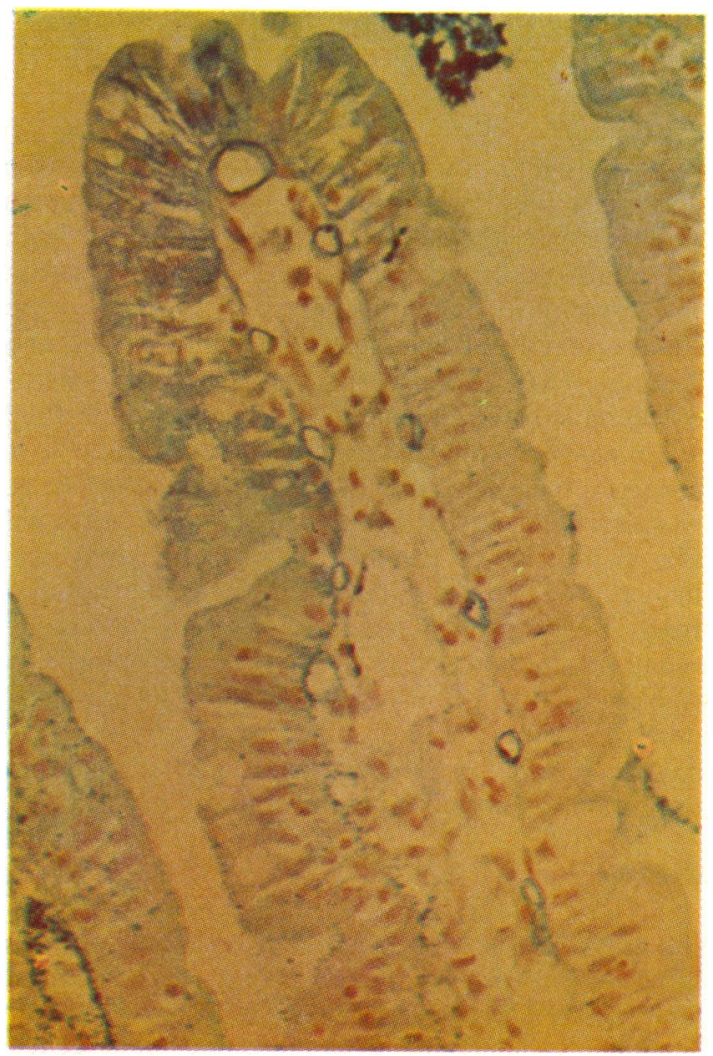

Fig 2 Perls' stain of a jejunal villus one minute after the injection into the mesenteric artery of ferrous sulphate. $\times 500$. 
junctional staining is well demonstrated in fig 3 which is a high-power view of a portion of a villus near the tip $\mathbf{3 0}$ seconds after the injection of ferric sulphate. Some villi showed no stain at all, presumably reflecting differences in regional blood flow after the injection, but those that did stain always showed the pattern demonstrated. Five minutes after injection staining persisted in the capillary basement membrane and in the cells at the tips, especially those at the extrusion zones. At 30 minutes a similar pattern was seen but there was also staining at the brush border region of all villous cells, possibly from iron which had passed through the mucosa. A similar picture was seen one hour after injection. Sections taken after the injection of Jectofer and Imferon showed no staining.

INTRALUMINAL IRON

After the intraluminal instillation of ferrous and ferric sulphate there was little staining demonstrated till one minute, after which time the brush border

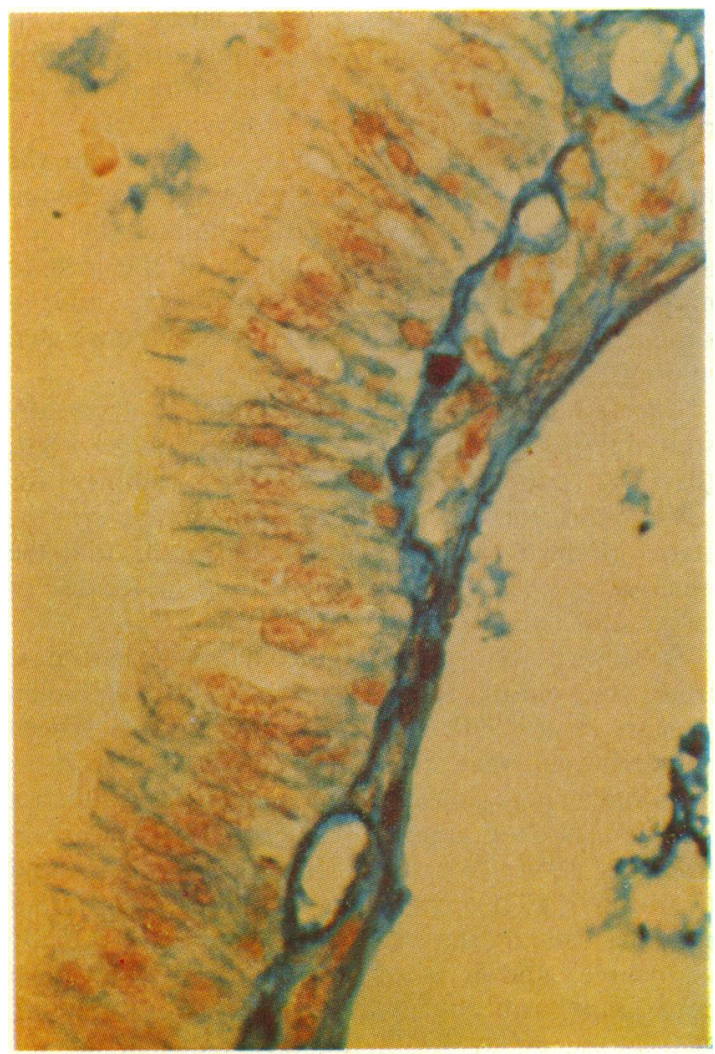

Fig 3 Perls' stain of portion of a villous tip 30 seconds after the injection into the mesenteric artery of ferric sulphate. $\times 800$. of all the villous cells became stained, possibly because of the mucopolysaccharide content of the glycocalyx. At five minutes the epithelial cells at the very tips of the villi showed staining and some iron was seen here at the junctional zone. The capillaries right at the tips also showed some stain. At 30 minutes some staining was observed in most of the epithelial cells on the upper third of the villi, the brush border throughout remained densely stained, and a similar picture remained up to one hour after instillation. Imferon and Jectofer showed no staining.

\section{ISCHAEMIC EXPERIMENTS}

Figure 4 demonstrates the mucosa one minute after the intraarterial injection of ferrous sulphate which had been injected after a 20-minute period of ischaemia. There is a patchy staining of iron throughout the mucosa and crypt cells also show staining. The critical period of ischaemia before such patchy changes occurred lay between 10 and 20 minutes.

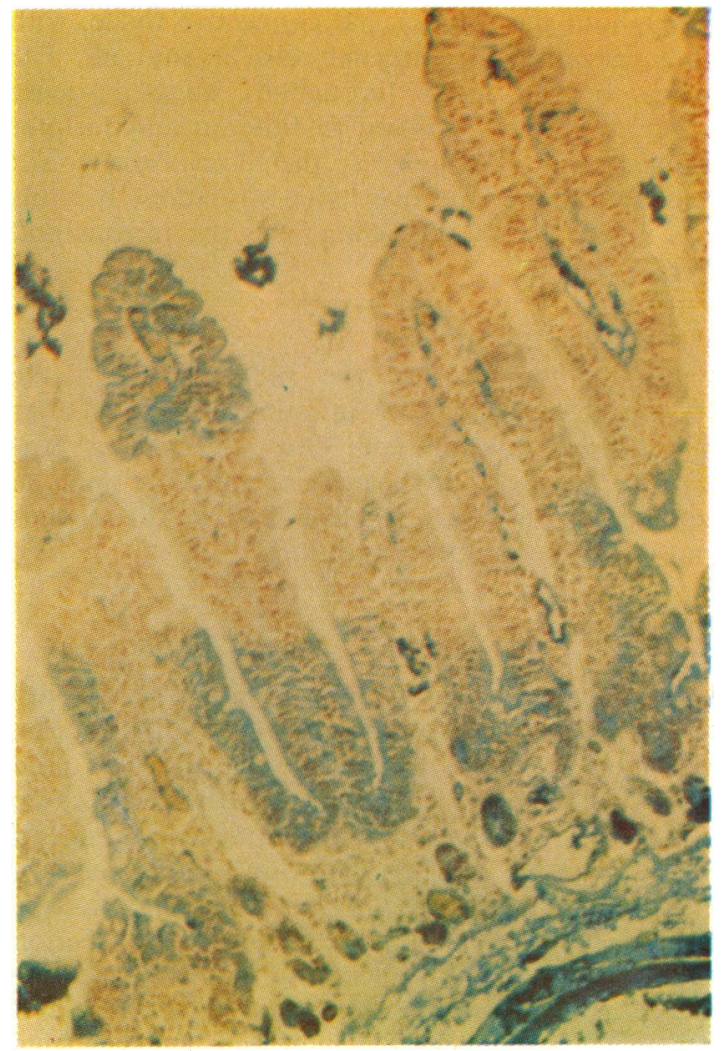

Fig 4 Perls' stain of jejunal mucosa subjected to 20 minutes' ischaemia one minute after the injection into the mesenteric artery of ferrous sulphate. $\times 160$. 


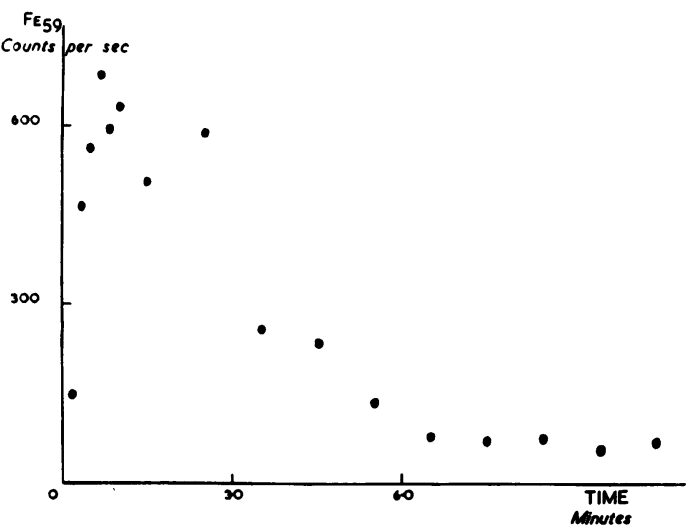

Fig 5 Radioactivity appearing in the jejunal lumen after the injection into the mesenteric artery of ${ }^{50} \mathrm{Fe}$.

FRESH FROZEN SECTIONS

Fresh frozen sections exposed to ferrous and ferric sulphate showed a diffuse uptake and staining throughout the mucosa and connective tissue, with no localization in epithelial cells at any site. The brush border layer throughout showed dense staining. Sections exposed to Imferon and. Jectofer showed slight staining of epithelial cells and goblet cells and also connective tissue structures, but this was much less marked than with the ferrous and ferric sulphate.

${ }^{50}$ FE EXPERIMENTS

Figure 5 demonstrates the radioactivity in the 10-second collections of intestinal perfusate after intraarterial injection of ${ }^{59} \mathrm{Fe}$, and shows how rapidly the iron permeates through the mucosa with maximum levels reached between 30 seconds and three minutes after injection.

\section{Discussion}

It is recognized that iron is rapidly bound onto chromosomes, centrosomes, mitochondria, nuclei, and other cytological structures, and iron is used in the field of mucosaccharide histochemistry because of the affinity of the ferric ion to free acid groups of mucosaccharide (Spicer and Sun, 1967; Tyrkkö, Häkkinen, and Rimpelä, 1968; Gad and Sylvén, 1969; Sorvari, 1972), and the Prussian blue reaction demonstrated in the present study almost certainly reflects iron bound in this way. Both ferric and ferrous sulphate showed staining with Prussian blue as the mucosa is known to convert rapidly the ferrous ion to the ferric form. It was theoretically possible that the pattern of staining demonstrated was due to the specific localization of mucosac- charides and other iron-binding substances in different parts of the mucosa rather than reflecting the pathway of iron excretion. However, the experiments on the fresh frozen sections failed to show such specific staining. The study therefore suggests that iron passes through the capillary wall and from there between epithelial cells where the ferric ion was shown to be concentrated within 10 seconds of injection. This was most marked at the upper parts of the villi and perhaps cells here shortly to become extruded are less closely in apposition. Shortly afterwards iron appears in the epithelial cells at the tips of the villi and then more diffusely throughout villous epithelial cells, though with the densest concentration always at the tips. These results seem to be at variance with those of Conrad and Crosby (1963), who demonstrated radioactivity by autoradiographic techniques after the injection of ${ }^{59} \mathrm{Fe}$ maximally in the crypt region. However, in their experiments the earliest biopsies were taken at two hours after injection and this probably represents the actual uptake of iron into epithelial cells as opposed to the present study which is concerned with the immediate direct permeability of the mucosa to iron as demonstrated by the rapid intraluminal appearance of injected ${ }^{59} \mathrm{Fe}$.

In the experiments where iron was put into the lumen the early staining was confined to the region of the brush border covering the whole of the villi, and probably represents the affinity of the iron to mucopolysaccharide in the glycocalyx, and a similar concentration in this area was demonstrated in the fresh frozen sections.

The failure of Imferon and Jectofer to show any staining was probably due to two factors. First, that the bound molecule had less affinity for tissue constituents, as was demonstrated by the stains of fresh frozen tissue, and secondly that the mucosa was less permeable to these molecules of larger molecular sizes.

Substances of varying molecular size can pass from the plasma out into the intestinal lumen (Loehry et al, 1970; Loehry, 1973), and it has been demonstrated that even macromolecules such as horseradish peroxidase may pass through the mucosa (Cornell, Walker, and Isselbacher, 1971; Hugon and Borgers, 1968; Warshaw, Walker, Cornell, and Isselbacher, 1971). The present study has suggested that the cells at the tips of the villi are especially permeable, and it is of interest that horseradish peroxidase has been demonstrated after intraluminal instillation, especially in the epithelial cells at the villous tips (Warshaw et al; 1971), and it was suggested that some passive diffusion of these macromolecules occurred at this site. In a study utilizing autoradiography after the intravenous 
injection of ${ }^{125} \mathrm{I}$ albumin Brooks and Dobbins (1972) demonstrated some activity between epithelial cells, especially at the villous tips.

Molecules passing from the blood out into the intestinal lumen first have to cross the capillary wall, but it seems unlikely that this presents a major barrier as substances as large as ferritin have been shown to cross here (Clementi and Palade, 1969). Having traversed the capillary and basement membrane three possible routes are open to the permeating molecules: they may pass between epithelial cells, through them, or through the gaps left by extruded cells at the tips of the villi. The evidence from the present study suggests that iron, at any rate, passes up between cells as far as the tight junction and also through cells, and that this process is maximal at the tips of the villi where the cells are reaching the end of their life span. It is of interest to speculate on the implications of this method of intestinal loss in disease states. In the present study it has been demonstrated how a short period of ischaemia renders cells that were previously relatively permeable able to take up iron, and it is possible that substances may pass into the lumen in small intestinal diseases by an increased permeability of disordered epithelial cells.

I am grateful to the Wessex Regional Board Re- search Committee for providing funds that have made this work possible.

References

Brooks, S. G., and Dobbins, W. O. (1972). Autoradiographic localization of $\mathrm{I}^{125}$ labeled albumin in the intestine of guinea pigs: a light and electron miroscopic study. Gastroenterology, 62, 1001-1012.

Clementi, F., and Palade, G. E. (1969). Intestinal capillaries. I. Permeability to peroxidase and ferritin. J. Cell Biol., 41, 33-58.

Conrad, M. E., Jr., and Crosby, W. H. (1963). Intestinal mucosal mechanisms controlling iron absorption. Blood, 22, 406-415.

Cornell, R., Walker, W. A., and Isselbacher, K. J. (1971). Small intestinal absorption of horseradish peroxidase. Lab. Invest., 25, 42-48.

Gad, A., and Sylven, B. (1969). On the nature of the high iron diamine method for sulfomucins. J. Histochem. Cytochem., 17, 156-160.

Hugon, J. S., and Borgers, M. (1968). Absorption of horseradish peroxidase by the mucosal cells of the duodenum of the mouse. I. The fasting animal. J. Histochem. Cytochem., 16, 229-236.

Loehry, C. A., Axon, A. T. R., Hilton, P. J., Hider, R. C., and Creamer, B. (1970). Permeability of the small intestine to substances of different molecular weight. Gut, 11, 466-470.

Loehry, C. A. (1973). Small intestinal permeability in animals and man. Part I. Small intestinal permeability in man. Gut, 14, 683-685.

Sorvari, T. E (1972). Binding of ferric ions to nuclei and other tissue sites in sections stained for sulfated mucosubstances by the high iron diamine method. Stain Technol., 47, 245-248.

Spicer, S. S., and Sun, D. C. H. (1967). Carbohydrate histochemistry of gastric epithelial secretions in dog. Ann. N.Y. Acad. Sci., 140, 762-783.

Tyrkkö, J., Hăkkinen, I., and Rimpelă, U. (1968). On the histochemical demonstration of sulphomucins in human and canine gastric epithelium. Brit. J. exp. Path., 49, 371-374.

Warshaw, A. L., Walker, W. A., Cornell, R., and Isselbacher, K. J. (1971). Small intestinal permeability to macromolecules. Lab. Invest., 25, 675-684. 